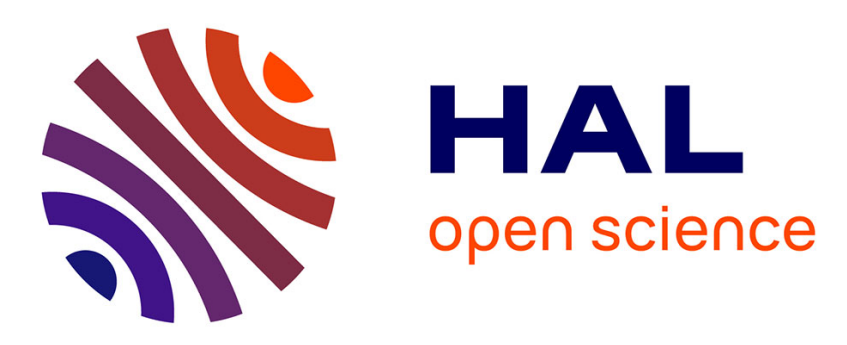

\title{
Some Support Considerations in the Asymptotic Optimality of Two-Scale Controlled PDMP
}

\author{
D Goreac, Oana Silvia Serea
}

\section{To cite this version:}

D Goreac, Oana Silvia Serea. Some Support Considerations in the Asymptotic Optimality of TwoScale Controlled PDMP. Viorel Barbu ("Al I Cuza" University of Iaşi, Romania \& Romanian Academy, Romania), Cătălin Lefter ("Al I Cuza" University of Iaşi, Romania \& Romanian Academy, Romania), Ioan I Vrabie ("Al I Cuza" University of Iaşi, Romania \& Romanian Academy, Romania). New Trends in Differential Equations, Control Theory and Optimization, World Scientific, pp.155-171, 2016, 10.1142/9789813142862_0012. hal-01359795

\section{HAL Id: hal-01359795 https://hal.science/hal-01359795}

Submitted on 4 Sep 2016

HAL is a multi-disciplinary open access archive for the deposit and dissemination of scientific research documents, whether they are published or not. The documents may come from teaching and research institutions in France or abroad, or from public or private research centers.
L'archive ouverte pluridisciplinaire HAL, est destinée au dépôt et à la diffusion de documents scientifiques de niveau recherche, publiés ou non, émanant des établissements d'enseignement et de recherche français ou étrangers, des laboratoires publics ou privés. 


\title{
Some Support Considerations in the Asymptotic Optimality of Two-Scales Controlled PDMP
}

\author{
D. Goreac \\ Université Paris-Est, LAMA (UMR 8050), UPEMLV, UPEC, CNRS, \\ F-77454, Marne-la-Vallée, France \\ E-mail: Dan.Goreac@u-pem.fr \\ O. S. Serea \\ Université de Perpignan Via Domitia, \\ Laboratoire de Mathématiques et de Physique, EA 4217, \\ F-66860, Perpignan, France \\ E-mail: oana-silvia.serea@univ-perp.fr (corresponding author)
}

\begin{abstract}
The aim of this short note is to give a linear programming approach to optimality conditions (expressed through support criteria) in control problems with piecewise deterministic Markov dynamics. Two classes are considered: classical, discounted control problems and asymptotic problems associated to two-scales (perturbed) systems.
\end{abstract}

\section{Introduction}

In this short note, we wish to show how linear programming methods may be used to infer support conditions for optimality for classical control problems for piecewise deterministic Markov processes (PDMP) and asymptotic control problems for two-scales PDMP.

We start by recalling the definition of a controlled PDMPs and by stating standard assumptions in Section 2. In Section 3, we deal with classical control problems with discounted cost and PDMP dynamics. We begin with recalling the linearized formulations (primal and dual) of discounted, infinite-horizon control problems. In this context, the dual formulation gives a reliable intuition on the structure of the support for the optimal measures. We briefly explain the notion of optimal pair and, whenever such pair exists, we explicitly give a support condition. In the general case, when the optimal value is not given by a regular function, we work with the optimality candidates using as regular function, the approximating functions obtained by the "shaking of coefficients" method in ${ }^{1}$. The 
support will then involve inner or outer limits of such sets.

In Section 4, we deal with control problems associated to two-scales PDMP. This is very much inspired by the literature on singularly perturbed systems (e.g. ${ }^{2-7}$ ). This kind of singular-perturbation problem is particularly challenging because, in many cases, one fails to identify the limit dynamics. This is the case even for simpler systems without jumps. Thus, a fortiori, one has difficulties in characterizing the asymptotic optimal controls. However, in particular cases, the value functions associated to the perturbed problem uniformly converge to the same value as the one obtained for the limit system. Moreover, the speed of convergence can be made explicit. We do not aim in this short note at giving explicit conditions to have this type of convergence. This makes the object of an ongoing research exploiting non-expansive properties for temperate virus inspired by $^{8}$. The conditions are intended in the spirit of ${ }^{9}$ Corollaries 3.4 and 4.3 (for Brownian setting). For already existing conditions of convergence, the interested reader is invited to consult the paper ${ }^{6}$ or ${ }^{3}$ and references therein. We use a double net (regularizations of the value functions associated to fixed scaling parameter) first to give a convenient dual asymptotic formulation and, second, to infer support conditions on the optimal measures. This is done using the intuitions already mentioned in Section 3.

\section{Controlled Piecewise Deterministic Processes and Standing Assumptions}

We consider $U$ to be a compact metric space (the control space) and $\mathbb{R}^{N}$ be the state space, for some $N \geq 1$.

Piecewise deterministic control processes have first been introduced by Davis $^{10}$ (see also ${ }^{11,12}$. They can be summarized by a characteristic triplet: a vector field $f: \mathbb{R}^{N} \times U \rightarrow \mathbb{R}^{N}$ that determines the motion between two consecutive jumps, a jump rate $\lambda: \mathbb{R}^{N} \times U \rightarrow \mathbb{R}_{+}$and a transition measure $Q: \mathbb{R}^{N} \times U \times \mathcal{B}\left(\mathbb{R}^{N}\right) \rightarrow \mathcal{P}\left(\mathbb{R}^{N}\right)$. Here $\mathcal{B}\left(\mathbb{R}^{N}\right)$ is the Borel $\sigma$-field on $\mathbb{R}^{N}$ and $\mathcal{P}\left(\mathbb{R}^{N}\right)$ stands for the family of probability measures on $\mathbb{R}^{N}$. For every $A \in \mathcal{B}\left(\mathbb{R}^{N}\right)$, the function $(u, x) \mapsto Q(x, u, A)$ is assumed to be measurable and, for every $(x, u) \in \mathbb{R}^{N} \times U, Q(x, u,\{x\})=0$.

Whenever $u \in \mathbb{L}^{0}\left(\mathbb{R}^{N} \times \mathbb{R}_{+} ; U\right)$ ( $u$ is a Borel measurable function) and $\left(t_{0}, x_{0}\right) \in \mathbb{R}_{+} \times \mathbb{R}^{N}$, we consider the ordinary differential equation

$$
\left\{\begin{array}{l}
d \Phi_{t}^{t_{0}, x_{0}, u}=f\left(\Phi_{t}^{t_{0}, x_{0}, u}, u\left(x_{0}, t-t_{0}\right)\right) d t, t \geq t_{0}, \\
\Phi_{t_{0}}^{t_{0}, x_{0}, u}=x_{0}
\end{array}\right.
$$

We choose the first jump time $T_{1}$ such that the jump rate 
$\lambda\left(\Phi_{t}^{0, x_{0}, u}, u\left(x_{0}, t\right)\right)$ satisfies

$$
\mathbb{P}\left(T_{1} \geq t\right)=\exp \left(-\int_{0}^{t} \lambda\left(\Phi_{s}^{0, x_{0}, u}, u\left(x_{0}, s\right)\right) d s\right) .
$$

The controlled piecewise deterministic Markov processes (PDMP) is defined by

$$
X_{t}^{x_{0}, u}=\Phi_{t}^{0, x_{0}, u}, \text { if } t \in\left[0, T_{1}\right) .
$$

The post-jump location $Y_{1}$ has $Q\left(\Phi_{\tau}^{0, x_{0}, u}, u\left(x_{0}, \tau\right), \cdot\right)$ as conditional distribution given $T_{1}=\tau$. Starting from $Y_{1}$ at time $T_{1}$, we select the inter-jump time $T_{2}-T_{1}$ such that

$$
\mathbb{P}\left(T_{2}-T_{1} \geq t / T_{1}, Y_{1}\right)=\exp \left(-\int_{T_{1}}^{T_{1}+t} \lambda\left(\Phi_{s}^{T_{1}, Y_{1}, u}, u\left(Y_{1}, s-T_{1}\right)\right) d s\right) .
$$

We set

$$
X_{t}^{x_{0}, u}=\Phi_{t}^{T_{1}, Y_{1}, u}, \text { if } t \in\left[T_{1}, T_{2}\right) .
$$

The post-jump location $Y_{2}$ satisfies

$$
\mathbb{P}\left(Y_{2} \in A / T_{2}, T_{1}, Y_{1}\right)=Q\left(\Phi_{T_{2}}^{T_{1}, Y_{1}, u}, u\left(Y_{1}, T_{2}-T_{1}\right), A\right),
$$

for all Borel set $A \subset \mathbb{R}^{N}$. And so on.

\subsection{Standing Assumptions}

Throughout the paper, unless stated otherwise, we assume the following:

(A1) The function $f: \mathbb{R}^{N} \times U \longrightarrow \mathbb{R}^{N}$ is uniformly continuous on $\mathbb{R}^{N} \times U$ and there exists a positive real constant $C>0$ such that

$$
|f(x, u)-f(y, u)| \leq C|x-y| \text {, and }|f(x, u)| \leq C,
$$

for all $x, y \in \mathbb{R}^{N}$ and all $u \in U$.

(A2) The function $\lambda: \mathbb{R}^{N} \times U \longrightarrow \mathbb{R}_{+}$is uniformly continuous on $\mathbb{R}^{N} \times U$ and there exists a positive real constant $C>0$ such that

$$
|\lambda(x, u)-\lambda(y, u)| \leq C|x-y|, \text { and } \lambda(x, u) \leq C,
$$

for all $x, y \in \mathbb{R}^{N}$ and all $u \in U$.

(A3) For each bounded uniformly continuous function $h \in B U C\left(\mathbb{R}^{N}\right)$, there exists a continuous function $\eta_{h}: \mathbb{R} \longrightarrow \mathbb{R}$ such that $\eta_{h}(0)=0$ and

$$
\sup _{u \in U}\left|\int_{\mathbb{R}^{N}} h(z) Q(x, u, d z)-\int_{\mathbb{R}^{N}} h(z) Q(y, u, d z)\right| \leq \eta_{h}(|x-y|) .
$$


We will ask that $\eta$ should only depend on the continuity modulus and bound of $h$ but not of $h$ itself. of $\mathbb{R}^{N}$,

(A4) For every $x \in \mathbb{R}^{N}$ and every decreasing sequence $\left(\Gamma_{n}\right)_{n \geq 0}$ of subsets

$$
\inf _{n \geq 0} \sup _{u \in U} Q\left(x, u, \Gamma_{n}\right)=\sup _{u \in U} Q\left(x, u, \cap_{n} \Gamma_{n}\right) .
$$

Remark 2.1. The assumption A3 can be somewhat weakened by imposing

(A3') For each bounded uniformly continuous function $h \in B U C\left(\mathbb{R}^{N}\right)$, there exists a continuous function $\eta_{h}: \mathbb{R} \longrightarrow \mathbb{R}$ such that $\eta_{h}(0)=0$ and

$\sup _{u \in U}\left|\lambda(x, u) \int_{\mathbb{R}^{N}} h(z) Q(x, u, d z)-\lambda(y, u) \int_{\mathbb{R}^{N}} h(z) Q(y, u, d z)\right| \leq \eta_{h}(|x-y|)$.

It is obvious that whenever one assumes (A3) and $\lambda(\cdot)$ is bounded, the assumption A3' holds true.

To simplify the arguments, we assume the following invariance condition to hold true. There exists a compact set $\mathbb{K}$ such that, for every $x \in \partial \mathbb{K}$, every $p \in N_{\mathbb{K}}(x)$ and every $u \in U$, one has

$$
\langle f(x, u), p\rangle+\lambda(x, u) Q\left(x, u, \mathbb{K}^{c}\right) \leq 0
$$

Here, $\mathbb{K}^{c}$ stands for $\mathbb{R}^{N} \backslash \mathbb{K}$, while $N_{\mathbb{K}}(x)$ stands for the normal cone to $\mathbb{K}$ at $x$, denoted by $N_{\mathbb{K}}(x)$ and is defined as

$N_{\mathbb{K}}(x)=\left\{p \in \mathbb{R}^{N}: \forall \varepsilon>0, \exists \eta>0\right.$ such that $\left.\forall y \in \mathbb{K} \cap \bar{B}(x, \eta),\langle p, y-x\rangle \leq \varepsilon|y-x|\right\}$

We recall that $\bar{B}(x, \eta)=\left\{y \in \mathbb{R}^{N}:|y-x| \leq \eta\right\}$. This assumption implies that the set $\mathbb{K}$ is invariant with respect to the piecewise deterministic Markov dynamics (every trajectory starting from a point of $\mathbb{K}$ remains in $\mathbb{K}$ almost surely). For further details on invariance of piecewise deterministic Markov processes, the reader is referred to ${ }^{13}$.

Remark 2.2. This condition implies that if $\mathbb{K}$ is invariant w.r.t. the PDMP with characteristic triplet $(f, \lambda, Q)$, it also holds true for $\left(\delta f, \delta^{\prime} \lambda, \frac{\delta}{\delta^{\prime}} Q\right)$, for all $\delta, \delta^{\prime}>0$. 


\section{An LP Approach to Optimality in Classical Control Problems With PDMP Dynamics}

\subsection{Linear Programming Tools for PDMP}

Given a Lipschitz-continuous, bounded cost functional $g: \mathbb{R}^{N} \longrightarrow \mathbb{R}$, we introduce the value function by setting

$$
v_{g}(x):=\inf _{u} \mathbb{E}\left[\int_{0}^{\infty} e^{-t} g\left(X_{t}^{x, u}\right) d t\right], \text { for all } x \in \mathbb{R}^{N}
$$

The following results are taken from ${ }^{14}$.

We denote by $\mathcal{P}\left(\mathbb{R}^{N} \times U\right)$ the set of all probability measures on $\mathbb{R}^{N} \times U$ and by

$\Theta(x)=\left\{\gamma \in \mathcal{P}\left(\mathbb{R}^{N} \times U\right): \forall \phi \in C_{b}^{1}\left(\mathbb{R}^{N}\right): \int_{\mathbb{R}^{N} \times U}\left(\mathcal{U}^{u} \phi(y)+\phi(x)-\phi(y)\right) \gamma(d y, d u)=0\right\}$.

Here, $\mathcal{U}^{u}$ stands for the classical infinitesimal generator (associated to frozen control parameter $u \in U$ ) given by

$$
\mathcal{U}^{u} \phi(y)=\langle\nabla \phi(y), f(y, u)\rangle+\lambda(y, u) \int_{\mathbb{R}^{N}}(\phi(z)-\phi(y)) Q(y, u, d z),
$$

for all $u \in U, \phi \in C_{b}^{1}\left(\mathbb{R}^{N}\right)$, and all $y \in \mathbb{R}^{N}$. We recall that $C_{b}^{1}\left(\mathbb{R}^{N}\right)$ stands for the class of bounded, differentiable functions $\phi: \mathbb{R}^{N} \longrightarrow \mathbb{R}$ for which the first order derivatives are continuous and bounded. With respect to this closed, convex set $\Theta$, one introduces the linear (primal and dual) value functions

$$
\Lambda_{g}(x):=\inf _{\gamma \in \Theta(x)} \int_{\mathbb{R}^{N} \times U} g(y) \gamma(d y, d u)
$$

and

$$
\Lambda_{g}^{*}(x)=\sup \left\{\begin{array}{c}
\mu \in \mathbb{R}: \quad \exists \varphi \in C_{b}^{1}\left(\mathbb{R}^{N}\right) \text { such that } \forall(y, u) \in \mathbb{R}^{N} \times U, \\
\mu \leq \mathcal{U}^{u} \varphi(y)+g(y)+(\varphi(x)-\varphi(y))
\end{array}\right\},
$$

for all $x \in \mathbb{R}^{N}$. The link between the initial value function and the linear values is given by ${ }^{14}$ Theorem 7 .

Theorem 3.1 ( ${ }^{14}$ Theorem 7). For every $x \in \mathbb{R}^{N}$, the equality

$$
v_{g}(x)=\Lambda_{g}(x)=\Lambda_{g}^{*}(x)
$$

holds true. 
Remark 3.1. As a by-product, the set $\Theta(x)$ can be seen as the closed convex hull of occupation measures associated to the trajectories of the process. In particular, if the assumption (I) holds true, then, for every $x \in \mathbb{K}$, any measure $\gamma \in \Theta(x)$ has its support in $\mathbb{K} \times U$. Moreover, under the assumption (I), by introducing

$\Lambda_{g, \mathbb{K}}^{*}(x):=\sup \left\{\mu \in \mathbb{R}: \exists \varphi \in C_{b}^{1}\left(\mathbb{R}^{N}\right)\right.$ such that $\left.\forall(y, u) \in \mathbb{K} \times U, \mu \leq \mathcal{U}^{u} \varphi(y)+g(y)+(\varphi(x)-\varphi(y))\right\}$ one gets

$$
\Lambda_{g}(x) \geq \Lambda_{g, \mathbb{K}}^{*}(x) \geq \Lambda_{g}^{*}(x) .
$$

Hence, $\Lambda_{g, \mathbb{K}}^{*}(x)=\Lambda_{g}^{*}(x)$.

A careful look at the proof of the ${ }^{14}$ Theorem 7 and ${ }^{13}$ Theorem 3.6 yields the following Krylov-type result.

Lemma 3.1. There exists a decreasing function (only depending on the continuity moduli of $Q$ and $g$ but not on the functions themselves) $\eta$ : $\mathbb{R}_{+} \longrightarrow \mathbb{R}_{+}$that satisfies $\lim _{\delta \rightarrow 0} \eta(\delta)=0$ and a family of $C_{b}^{1}$ functions $V^{\delta}$ such that

$$
V_{g}^{\delta}(x)-g(x)+H\left(x, \nabla V_{g}^{\delta}(x), V_{g}^{\delta}\right) \leq 0,
$$

for all $x \in \mathbb{R}^{N}$, where the Hamiltonian $H$ is given by

$H(x, p, \psi)=\sup _{u \in U}\left\{-\langle f(x, u), p\rangle-\lambda(x, u) \int_{\mathbb{R}^{N}}(\psi(z)-\psi(x)) Q(x, u, d z)\right\}$.

and such that

$$
\sup _{x \in \mathbb{R}^{N}}\left|V_{g}^{\delta}(x)-v_{g}(x)\right| \leq C \eta(C \delta),
$$

for all $\delta>0$. The constant $C$ can be chosen as the maximum between the Lipschitz norms of $f$ and $\lambda$.

The function $\eta$ corresponding to a continuity modulus can be chosen at best of Lipschitz-continuity type (i.e. $\eta(x)=O(x)$ as $x \rightarrow 0$ ).

\subsection{Support Conditions for Optimality}

Due to Theorem 3.1 (the dual formulation $\Lambda^{*}$ ) and to Remark 3.1, in order to compute $v_{g}$ for arguments in $\mathbb{K}$, one tries to maximize, over test functions $\varphi \in C_{b}^{1}\left(\mathbb{R}^{N}\right)$, the infimum

$$
\inf _{(y, u) \in \mathbb{K} \times U} \mathcal{U}^{u} \varphi(y)+g(y)+(\varphi(x)-\varphi(y)) .
$$


Thus, it is natural to introduce the set

$D_{g}(x):=\left\{(\mu, \varphi) \in \mathbb{R} \times C_{b}^{1}\left(\mathbb{R}^{N}\right)\right.$ s.t. $\left.\mu=\inf _{(y, u) \in \mathbb{K} \times U} \mathcal{U}^{u} \varphi(y)+g(y)+(\varphi(x)-\varphi(y))\right\}$

for all $x \in \mathbb{K}$. The set $D_{g}(x)$ is well defined and non-empty (consequence of the assumptions on the characteristic triplet and cost function $g$ ). One can alternatively write the value function(s) as

$$
v_{g}(x)=\sup \left\{\mu,(\mu, \varphi) \in D_{g}(x)\right\},
$$

for all $x \in \mathbb{K}$. We introduce the following.

Definition 3.1. Whenever $x \in \mathbb{K}$, the couple $(\bar{\mu}, \bar{\varphi}) \in D_{g}(x)$ is an optimal pair if it satisfies $v_{g}(x)=\bar{\mu}$. If such pair exists, we denote by

$\Omega_{g,(\bar{\mu}, \bar{\varphi})}(x):=\left\{(y, u) \in \mathbb{K} \times U\right.$, s.t. $\left.\bar{\mu}=\mathcal{U}^{u} \bar{\varphi}(y)+g(y)+(\bar{\varphi}(x)-\bar{\varphi}(y))\right\}$.

As one easily understands, optimal measures have their support in such sets (should they exist). The problem is that optimal pairs do not always exist. A simple case when they do exist is when the value function itself belongs to $C_{b}^{1}\left(\mathbb{R}^{N}\right)$ (one just takes $\bar{\varphi}=v_{g}$ and uses the subsolution property for the associated HJB equation (see ${ }^{14}$ Theorem 18) to infer

$$
v_{g}(x) \leq \mathcal{U}^{u} v_{g}(y)+g(y)+\left(v_{g}(x)-v_{g}(y)\right),
$$

for all $(y, u) \in \mathbb{K} \times U$. In this case compactness of $\mathbb{K} \times U$ implies that $\Omega_{g,(\bar{\mu}, \bar{\varphi})}(x)$ is non empty.)

Proposition 3.1. Let $x \in \mathbb{K}$ and assume that $(\bar{\mu}, \bar{\varphi}) \in D_{g}(x)$ is an optimal pair. Then, $\gamma \in \Theta(x)$ is optimal for $v_{g}(x)$ if and only if $\gamma\left(\Omega_{g,(\bar{\mu}, \bar{\varphi})}(x)\right)=$ 1.

Proof. To prove the necessity of the support condition, let us consider $\gamma \in \Theta(x)$ which is optimal. It follows, by optimality and the definition of $\Theta(x)$, that

$v_{g}(x)=\int_{\mathbb{K} \times \mathbb{U}} g(y) \gamma(d y d u)=\int_{\mathbb{K} \times \mathbb{U}}\left(\mathcal{U}^{u} \bar{\varphi}(y)+g(y)+(\bar{\varphi}(x)-\bar{\varphi}(y))\right) \gamma(d y d u)$.

Then, the definition of $D_{g}(x)$ implies that $v_{g}(x) \geq \bar{\mu}$ and the equality holds true if and only if

$$
\mathcal{U}^{u} \bar{\varphi}(y)+g(y)+(\bar{\varphi}(x)-\bar{\varphi}(y))=\bar{\mu},
$$


$\gamma$-almost surely (or, equivalently, $\gamma\left(\Omega_{g,(\bar{\mu}, \bar{\varphi})}(x)\right)=1$ ). But the optimality of the pair $(\bar{\mu}, \bar{\varphi})$ can only happen if $v_{g}(x)=\bar{\mu}$ and, thus, the conclusion follows.

For sufficiency, one writes

$$
\mathcal{U}^{u} \bar{\varphi}(y)+g(y)+(\bar{\varphi}(x)-\bar{\varphi}(y))=\bar{\mu}=v_{g}(x),
$$

$\gamma$-almost surely and integrates on $\mathbb{K} \times \mathbb{U}$ w.r.t. $\gamma$ to get optimality.

In general, one simply picks the test functions $V_{g}^{\delta}$ and $\mu^{\delta}$ such that $\left(\mu^{\delta}, V_{g}^{\delta}\right) \in D_{g}(x)$. If among these functions an optimal pair does not exist (thus implying the existence of a strictly increasing sequence denoted, by abuse of notation, $\left(\mu^{n}\right)_{n \geq 1}$ such that $\left.\sup _{n} \mu^{n}=v_{g}(x)\right)$, one introduces an approximate set

$\Omega_{g}^{n}(x):=\left\{(y, u) \in \mathbb{K} \times U\right.$, s.t. $\left.v_{g}(x)+\eta\left(\left(v_{g}(x)-\mu^{n}\right)^{\frac{1}{2}}\right) \geq \mathcal{U}^{u} \bar{\varphi}(y)+g(y)+(\bar{\varphi}(x)-\bar{\varphi}(y))\right\}$.

and the usual (inner ant outer) limit sets

$$
\begin{aligned}
& \Omega_{g}^{\text {in }}(x):=\liminf _{n \rightarrow \infty} \Omega_{g}^{n}(x)=\underset{n \geq 1}{\cup} \cap_{n \geq n} \Omega_{g}^{k}(x), \Omega_{g}^{\text {out }}(x):=\limsup _{n \rightarrow \infty} \Omega_{g}^{n}(x)=\underset{n \geq 1}{\cap} \underset{k \geq n}{\cup} \Omega_{g}^{k}(x), \\
& \Omega_{g}^{\text {out }, c l}(x):=\bigcap_{n \geq 1} c l\left(\underset{k \geq n}{\cup} \Omega_{g}^{k}(x)\right),
\end{aligned}
$$

where $c l$ is the usual Kuratowski closure operator. Again by abuse of notation, we let $V_{g}^{n}$ be such that $\left(\mu^{n}, V_{g}^{n}\right) \in D_{g}(x)$. The support of optimal measures is characterized as follows.

\section{Proposition 3.2.}

(i) For every $x \in \mathbb{K}$, if $\gamma \in \Theta(x)$ is an optimal measure, then its support is contained in $\Omega_{g}^{\text {out }}(x) \subset \Omega_{g}^{\text {out }, c l}(x)$. In particular, when the limit of the sets exists (i.e. $\Omega_{g}^{\text {in }}(x)=\Omega_{g}^{\text {out }}(x)$ ), one has

$$
\sup _{n \geq 1}\left(\underset{k \geq n}{\cap} \Omega_{g}^{k}(x)\right)=1 .
$$

(ii) As a (partial) converse, for $x \in \mathbb{K}$, if $\gamma \in \Theta(x)$ is such that $\max _{n \geq 1}$ $\gamma\left(\underset{k \geq n}{\cap} \Omega_{g}^{k}(x)\right)=1$, then $\gamma$ is optimal.

Proof. (i) When $\gamma \in \Theta(x)$ is optimal, one has

$$
\begin{aligned}
v_{g}(x) & =\int_{\mathbb{K} \times \mathbb{U}} g(y) \gamma(d y d u)=\int_{\mathbb{K} \times \mathbb{U}}\left(\mathcal{U}^{u} V_{g}^{k}(y)+g(y)+\left(V_{g}^{k}(x)-V_{g}^{k}(y)\right)\right) \gamma(d y d u) \\
& \geq \int_{\mathbb{K} \times \mathbb{U}}\left(\mu^{k} 1_{\Omega_{g}^{k}(x)}+\left[v_{g}(x)+\eta\left(\left(v_{g}(x)-\mu^{k}\right)^{\frac{1}{2}}\right)\right] 1_{(\mathbb{K} \times \mathbb{U}) \backslash \Omega_{g}^{k}(x)}\right) \gamma(d y d u)
\end{aligned}
$$


for all $k \geq 1$. It follows that

$$
\left(\eta\left(\left(v_{g}(x)-\mu^{k}\right)^{\frac{1}{2}}\right)+1\right) \gamma\left(\Omega_{g}^{k}(x)\right) \geq\left(\frac{v_{g}(x)-\mu^{k}}{\eta\left(\left(v_{g}(x)-\mu^{k}\right)^{\frac{1}{2}}\right)}+1\right) \gamma\left(\Omega_{g}^{k}(x)\right) \geq 1
$$

One passes to $\limsup _{k \rightarrow \infty}$ to get $\gamma\left(\Omega_{g}^{\text {out }}(x)\right)=1$ and the proof is complete.

(ii) Whenever $\gamma\left(\underset{k \geq n_{0}}{\cap} \Omega_{g}^{k}(x)\right)=1$, it follows that $\gamma\left(\Omega_{g}^{k}(x)\right)=1$, for all $k \geq n_{0}$. Then, one simply writes down

$$
\begin{aligned}
v_{g}(x) & \leq \int_{\mathbb{K} \times \mathbb{U}} g(y) \gamma(d y d u)=\int_{\mathbb{K} \times \mathbb{U}}\left(\mathcal{U}^{u} V_{g}^{k}(y)+g(y)+\left(V_{g}^{k}(x)-V_{g}^{k}(y)\right)\right) \gamma(d y d u) \\
& \leq v_{g}(x)+\eta\left(\left(v_{g}(x)-\mu^{k}\right)^{\frac{1}{2}}\right),
\end{aligned}
$$

for all $k \geq n_{0}$. Passing to the limit as $k \rightarrow \infty$, one infers that $\gamma \in \Theta(x)$ is optimal.

\section{Two-Scale Systems. Asymptotic Optimality}

\subsection{A PDMP Description of Scales}

We consider a piecewise deterministic model set on $\mathbb{R}^{N+M}$ in which the second component (say regime) is faster than the first one and so is the frequency of its jumps. (To have a simple example, think of this second component as a discrete one rapidly toggling between the elements of the Euclidian basis of $\mathbb{R}^{M}$ and specifying different regime evolutions for some proteins). At any point, jumps can occur by:

- either changing the regime but, to express in terms of the previous example, keep continuous protein level;

- or, within the same regime, a slower (and much more rare) jump (say transcription) occurs and it is seen at protein level.

This amounts to considering a PDMP governed by a controlled characteristic triple $\left(f^{\varepsilon}, \lambda^{\varepsilon}, Q^{\varepsilon}\right)$ such that

$$
\begin{aligned}
& f^{\varepsilon}=\left(f_{1}, \frac{1}{\varepsilon} f_{2}\right), \lambda^{\varepsilon}(y, u)=\lambda_{1}(y, u)+\frac{1}{\varepsilon} \lambda_{2}(y, u) \\
& Q^{\varepsilon}\left(\left(y_{1}, y_{2}\right), u, d z_{1} d z_{2}\right)=\frac{\lambda_{1}(y, u)}{\lambda^{\varepsilon}(y, u)} Q_{1}\left(y_{2}\right)\left(y_{1}, u, d z_{1}\right)+\frac{\lambda_{2}(y, u)}{\varepsilon \lambda^{\varepsilon}(y, u)} Q_{2}\left(y_{1}\right)\left(y_{2}, u, d z_{2}\right) .
\end{aligned}
$$

Here, $f_{1}: \mathbb{R}^{N+M} \times U \longrightarrow \mathbb{R}^{N}, f_{2}: \mathbb{R}^{N+M} \times U \longrightarrow \mathbb{R}^{M}$ are bounded, uniformly continuous and Lipschitz-continuous in space, uniformly w.r.t. the control (i.e. satisfy the same assumptions as in the classical context). Similar regularity is assumed for $\lambda_{1,2}$ and $Q$. Moreover, we assume the 
existence of invariant sets $\mathbb{K}_{1}$ and $\mathbb{K}_{2}$ for $\left(f_{1}, \lambda_{1}\left(\cdot, y_{2}\right), Q_{1}\left(y_{2}\right)\right)$, for all $y_{2} \in \mathbb{K}_{2}$ respectively $\left(f_{2}, \lambda_{2}\left(y_{1}, \cdot\right), Q_{2}\left(y_{1}\right)\right)$, for all $y_{1} \in \mathbb{K}_{1}$. We will also use the notation $Q_{1}\left(y_{1}, y_{2}, u, d z_{1}\right)$ for $Q_{1}\left(y_{2}\right)\left(y_{1}, u, d z_{1}\right)$.

This kind of singular-perturbation problem is particularly challenging because, in many cases, one fails to identify the limit dynamics. This is the case even for simpler systems without jumps. Thus, a fortiori, one has difficulties in characterizing the asymptotic optimal controls. However, in particular cases, the value functions associated to the perturbed problem uniformly converge to the same value as the one obtained for the limit system. Moreover, the speed of convergence can be made explicit.

We do not aim in this short note at giving explicit conditions to have this type of convergence. This makes the object of an ongoing research exploiting non-expansive properties for temperate virus inspired by ${ }^{8}$. The conditions are intended in the spirit of ${ }^{9}$ Corollaries 3.4 and 4.3 (for Brownian setting). For already existing conditions of convergence, the interested reader is invited to consult the paper ${ }^{6}$ or ${ }^{3}$ and references therein.

\subsection{Asymptotics of Linearized Formulations}

Inspired by the considerations on classical discounted control problems presented in the first part, we introduce the perturbed set of constraints

$$
\Theta^{\varepsilon}(x)=\left\{\begin{array}{c}
\gamma \in \mathcal{P}\left(\mathbb{R}^{N+M} \times U\right): \forall \phi \in C_{b}^{1}\left(\mathbb{R}^{N+M}\right), \\
\int_{\mathbb{R}^{N} \times U}\left(\mathcal{U}^{u ; \varepsilon} \phi(y)+\phi(x)-\phi(y)\right) \gamma(d y, d u)=0
\end{array}\right\},
$$

were $\mathcal{U}^{u . \varepsilon}$ stands for the infinitesimal generator associated to $\left(f^{\varepsilon}, \lambda^{\varepsilon}, Q^{\varepsilon}\right)$ and given by

$$
\begin{gathered}
\mathcal{U}^{u . \varepsilon} \phi\left(y_{1}, y_{2}\right)=\left\langle\nabla_{y_{1}} \phi(y), f_{1}(y, u)\right\rangle+\frac{1}{\varepsilon}\left\langle\nabla_{y_{2}} \phi(y), f_{2}(y, u)\right\rangle \\
\quad+\lambda_{1}(y, u) \int_{\mathbb{R}^{N}}\left(\phi\left(z, y_{2}\right)-\phi\left(y_{1}, y_{2}\right)\right) Q_{1}(y, u, d z) \\
\quad+\frac{1}{\varepsilon} \lambda_{2}(y, u) \int_{\mathbb{R}^{M}}\left(\phi\left(y_{1}, z\right)-\phi\left(y_{1}, y_{2}\right)\right) Q_{2}(y, u, d z)
\end{gathered}
$$

for all $u \in U, \phi \in C_{b}^{1}\left(\mathbb{R}^{N+M}\right)$, and all $y=\left(y_{1}, y_{2}\right) \in \mathbb{R}^{N+M}$. We consider a cost functional $g$ having the same regularity as in the first part, but defined on $\mathbb{R}^{N+M}$ and set

$$
v_{g}^{\varepsilon}(x):=\inf _{u} \mathbb{E}\left[\int_{0}^{\infty} e^{-t} g\left(X_{t}^{x, u ; \varepsilon}\right) d t\right], \text { for all } x \in \mathbb{R}^{N+M},
$$

where $X_{t}^{x, u ; \varepsilon}$ is the PDMP admitting the local characteristics $\left(f^{\varepsilon}, \lambda^{\varepsilon}, Q^{\varepsilon}\right)$ and constructed as described before. Moreover, we set

$$
\Lambda_{g}^{\varepsilon}(x):=\inf _{\gamma \in \Theta^{\varepsilon}(x)} \int_{\mathbb{R}^{N+M} \times U} g(y) \gamma(d y, d u)
$$


and $\Lambda_{g}^{\varepsilon *}(x):=\sup \left\{\begin{array}{c}\mu \in \mathbb{R}: \exists \varphi \in C_{b}^{1}\left(\mathbb{R}^{N+M}\right) \text { such that, } \forall(y, u) \in \mathbb{R}^{N+M} \times U, \\ \mu \leq \mathcal{U}^{u ; \varepsilon} \varphi(y)+g(y)+(\varphi(x)-\varphi(y))\end{array}\right\}$,

for all $x \in \mathbb{R}^{N+M}$. The arguments on classical control problems yield that the value function $v_{g}^{\varepsilon}(\cdot)$ associated to a regular cost $g$ can, alternatively, be given by

$$
v_{g}^{\varepsilon}(x)=\Lambda_{g}^{\varepsilon}(x)=\Lambda_{g}^{\varepsilon *}(x), \text { for all } x \in \mathbb{R}^{N+M} .
$$

It is natural, at this point, to set

$$
\Theta\left(y_{1}^{0}, y_{2}^{0}\right):=\liminf _{\varepsilon \rightarrow 0} \Theta^{\varepsilon}\left(y_{1}^{0}, y_{2}^{0}\right), \text { for }\left(y_{1}^{0}, y_{2}^{0}\right) \in \mathbb{R}^{N+M} .
$$

(This is the classical lower limit for sets with the usual weak* convergence of measures). We begin with some simple considerations on the structure of this set of constraints.

Proposition 4.1. The following inclusion holds true $\Theta\left(y_{1}^{0}, y_{2}^{0}\right) \subset\left\{\begin{array}{c}\gamma \in \mathcal{P}\left(\mathbb{R}^{M} \times \mathbb{R}^{N} \times U\right) \text { s.t. } \forall \psi \in C_{b}^{1}\left(\mathbb{R}^{N}\right) \text { and } \forall \phi \in C_{b}^{1}\left(\mathbb{R}^{N+M}\right), \\ \int_{\mathbb{R}^{N+M} \times U}\left[\mathcal{U}^{u .1, y_{2}} \psi\left(y_{1}\right)+g\left(y_{1}, y_{2}\right)+\left(-\psi\left(y_{1}\right)+\psi\left(y_{1}^{0}\right)\right)\right] \gamma\left(d y_{1} d y_{2} d u\right)=0 \text { and } \\ \int_{\mathbb{R}^{N+M} \times U}\left[\mathcal{U}^{u, 2} \phi\left(y_{1}, y_{2}\right)+g\left(y_{1}, y_{2}\right)+\left(-\phi\left(y_{1}, y_{2}\right)+\phi\left(y_{1}^{0}, y_{2}^{0}\right)\right)\right] \gamma\left(d y_{1} d y_{2} d u\right)=0\end{array}\right\}$, where

$\mathcal{U}^{u, 1, y_{2}} \psi\left(y_{1}\right):=\left\langle\nabla \psi\left(y_{1}\right), f_{1}\left(y_{1}, y_{2}, u\right)\right\rangle+\lambda_{1}\left(y_{1}, y_{2}, u\right) \int_{\mathbb{R}^{N}}\left(\psi(z)-\psi\left(y_{1}\right)\right) Q_{1}\left(y_{1}, y_{2}, u, d z\right)$ and $\mathcal{U}^{u, 2} \phi\left(y_{1}, y_{2}\right):=\left\langle\nabla_{y_{2}} \phi\left(y_{1}, y_{2}\right), f_{2}\left(y_{1}, y_{2}, u\right)\right\rangle+\lambda_{2}\left(y_{1}, y_{2}, u\right) \int_{\mathbb{R}^{M}}\left(\phi\left(y_{1}, z\right)-\phi\left(y_{1}, y_{2}\right)\right) Q_{1}\left(y_{1}, y_{2}, u, d z\right)$, for all $\phi \in C^{1}\left(\mathbb{R}^{N+M}\right), \psi \in C^{1}\left(\mathbb{R}^{N}\right)$ and all $\left(y_{1}, y_{2}\right) \in \mathbb{R}^{N} \times \mathbb{R}^{M}, u \in U$.

Proof. The proof is standard and will be omitted.

We define the linearized problem with respect to the new set of constraints

$$
\Lambda_{g}\left(y_{1}^{0}, y_{2}^{0}\right):=\inf _{\gamma \in \Theta\left(y_{1}^{0}, y_{2}^{0}\right)} \int_{\mathbb{R}^{N+M} \times U} g(z) \gamma(d z d u) .
$$




\subsection{First Main Result: An Asymptotic Formulation}

Throughout the remaining of the paper, unless stated otherwise, we assume that there exists a continuity modulus $\omega$ only depending on the bound and continuity modulus of the cost function $g$ and the bound and continuous moduli of the characteristics of the PDMP and some function $\xi \in C_{b}\left(\mathbb{R}^{N}\right)$ (which may depend on $g$ and $(f, \lambda, Q)$ ) such that

$$
\sup _{\left(y_{1}, y_{2}\right) \in \mathbb{K}=\mathbb{K}_{1} \times \mathbb{K}_{2}}\left|v_{g}^{\varepsilon}\left(y_{1}, y_{2}\right)-\xi\left(y_{1}\right)\right| \leq \omega(\varepsilon) .
$$

In the Brownian setting, for further details and conditions implying this kind of convergence, the reader is referred to ${ }^{9}$; see also ${ }^{15}$.

If $\delta>0$ and $v_{g}^{\varepsilon, \delta}$ is the value function associated with the "shaken" problem (i.e. in which $\varphi \in\{g, f, \lambda\}$ are replaced with $\varphi^{\delta}(x,(u, v)):=$ $\varphi(x+\delta v, u), v \in \mathbb{R}^{N+M},|v| \leq 1$ respectively $Q$ is replaced by $\left.Q^{\delta}(x,(u, v), d y)=\widetilde{Q}(x+v, u, d y+v)\right)$, under analogous assumptions, the inequality (17) holds true for some $\xi^{\delta}$ replacing $\xi$. Since $\omega$ only depends on the continuity moduli, the right-hand member is a generic $\omega$ independent of $\delta>0$. In particular,

$$
\left|v_{g}^{\varepsilon, \delta}\left(y_{1}, y_{2}\right)-v_{g}^{\varepsilon, \delta}\left(y_{1}, y_{2}^{\prime}\right)\right| \leq 2 \omega(\varepsilon),
$$

for all $y_{1} \in \mathbb{K}_{1}$ and all $y_{2}, y_{2}^{\prime} \in \mathbb{K}_{2}$. Now, let us consider $\left(\psi_{\delta}\right)_{\delta}$ to be a sequence of standard mollifiers $\psi_{\delta}(x):=\frac{1}{\delta^{M+N}} \psi\left(\frac{x}{\delta}\right), x \in \mathbb{R}^{N+M}, \delta>0$, where $\psi \in C^{\infty}\left(\mathbb{R}^{M+N}\right)$ is a positive function such that

$$
\operatorname{Supp}(\psi) \subset \bar{B}(0,1) \text { and } \int_{\mathbb{R}^{N+M}} \psi(x) d x=1 .
$$

Then, the convoluted functions $V_{g}^{\varepsilon, \delta}:=v_{g}^{\varepsilon, \delta} * \psi_{\delta}$ satisfy (with the modulus $\eta$ given in Lemma 3.1 and due to the inequality (17)):

$$
\begin{aligned}
& \left|V_{g}^{\varepsilon, \delta}\left(y_{1}, y_{2}\right)-v_{g}^{\varepsilon}\left(y_{1}, y_{2}\right)\right| \leq c_{0}\left(1+\frac{1}{\varepsilon}\right) \eta\left(c_{0}\left(1+\frac{1}{\varepsilon}\right) \delta\right), \\
& \left|V_{g}^{\varepsilon, \delta}\left(y_{1}, y_{2}\right)-V_{g}^{\varepsilon, \delta}\left(y_{1}, y_{2}^{\prime}\right)\right| \leq 2 c_{0}\left(1+\frac{1}{\varepsilon}\right) \eta\left(c_{0}\left(1+\frac{1}{\varepsilon}\right) \delta\right)+\left|v_{g}^{\varepsilon}\left(y_{1}, y_{2}\right)-\xi\left(y_{1}\right)\right| \\
& \leq 2 c_{0}\left(1+\frac{1}{\varepsilon}\right) \eta\left(c_{0}\left(1+\frac{1}{\varepsilon}\right) \delta\right)+\omega(\varepsilon),
\end{aligned}
$$

where $c_{0}$ is independent of $\delta$ and $\varepsilon$. Finally, by recalling that $\nabla V_{g}^{\varepsilon, \delta}=$ $\frac{1}{\delta} v_{g}^{\varepsilon, \delta} * \nabla \psi_{\delta}$, one gets

$$
\left|\nabla V_{g}^{\varepsilon, \delta}\left(y_{1}, y_{2}\right)-\nabla V_{g}^{\varepsilon, \delta}\left(y_{1}, y_{2}^{\prime}\right)\right| \leq \frac{1}{\delta} 2 \omega(\varepsilon) \text { and }\left\|\nabla V_{g}^{\varepsilon, \delta}\right\|_{\infty} \leq \frac{c_{0}}{\delta},
$$

for some constant $c_{0}>0$ independent of $\delta$ and $\varepsilon$. As consequence, one gets the following representation theorem. 
Theorem 4.1. Let us assume that the value functions for the perturbed systems converge uniformly to a function $\xi \in C_{b}\left(\mathbb{R}^{N}\right)$ (in the sense of (17)). Then the limit value function has the following representation (for all $y_{2}^{0} \in \mathbb{K}_{2}$ )

$$
\xi\left(y_{1}^{0}\right)=\Lambda_{g}\left(y_{1}^{0}, y_{2}^{0}\right),
$$

for all $y_{1}^{0} \in \mathbb{K}_{1}$. This common value is given by $\Lambda_{g}^{*}\left(y_{1}^{0}\right):=\sup \left\{\begin{array}{l}\eta \in \mathbb{R}: \exists \alpha \in C\left(\mathbb{R}_{+} ; \mathbb{R}_{+}\right), \lim _{\varepsilon \rightarrow 0} \alpha(\varepsilon)=0 \text { s.t. } \forall \varepsilon>0, \exists \varphi \in C_{b}^{1}\left(\mathbb{R}^{N+M}\right) \text { s.t. } \\ \sup _{y, y^{\prime} \in \mathbb{R}^{M}}\left\|\varphi(\cdot, y)-\varphi\left(\cdot, y^{\prime}\right)\right\|_{\infty} \leq \alpha(\varepsilon) \text { and s.t. } \forall(y, u) \in \mathbb{R}^{N+M} \times U, \\ \eta \leq \mathcal{U}^{u ; \varepsilon} \varphi\left(y_{1}, y_{2}\right)+g\left(y_{1}, y_{2}\right)+\left\|-\varphi\left(y_{1}, \cdot\right)\right\|_{\infty}+\left\|\varphi\left(y_{1}^{0}, \cdot\right)\right\|_{\infty}\end{array}\right\}$ for all $y_{1}^{0} \in \mathbb{K}_{1}$.

Proof. The first assertion is obvious by invoking the equality between the classical value $v_{g}^{\varepsilon}$ and its linearized formulation $\Lambda_{g}^{\varepsilon}$ and the construction of the asymptotic constraints set (16). We only need to prove that $\Lambda_{g}^{*}\left(y_{1}^{0}\right)$ gives the same object which is less obvious from simply passing to the limit in the dual formulations $\Lambda_{g}^{\varepsilon *}$ in (15). To this purpose, let us fix, for the time being, $\gamma \in \Theta\left(y_{1}^{0}, y_{2}^{0}\right)$ as the limit of some sequence $\gamma^{\varepsilon} \in \Theta^{\varepsilon}\left(y_{1}^{0}, y_{2}^{0}\right)$. Moreover, let us take

$$
\begin{aligned}
& \eta \in \mathbb{R}: \exists \alpha \in C\left(\mathbb{R}_{+} ; \mathbb{R}_{+}\right), \lim _{\varepsilon \rightarrow 0} \alpha(\varepsilon)=0 \text { s.t. } \forall \varepsilon>0, \exists \varphi \in C_{b}^{1}\left(\mathbb{R}^{N+M}\right) \text { s.t. } \\
& \sup _{y, y^{\prime} \in \mathbb{R}^{M}}\left\|\varphi(\cdot, y)-\varphi\left(\cdot, y^{\prime}\right)\right\|_{\infty} \leq \alpha(\varepsilon) \text { and s.t. } \forall(y, u) \in \mathbb{R}^{M} \times U, \\
& \eta \leq \mathcal{U}^{u ; \varepsilon} \varphi\left(y_{1}, y_{2}\right)+g\left(y_{1}, y_{2}\right)+\left\|-\varphi\left(y_{1}, \cdot\right)\right\|_{\infty}+\left\|\varphi\left(y_{1}^{0}, \cdot\right)\right\|_{\infty}
\end{aligned}
$$

It is clear that

$$
\eta \leq \mathcal{U}^{u ; \varepsilon} \varphi\left(y_{1}, y_{2}\right)+g\left(y_{1}, y_{2}\right)+\left(\varphi\left(y_{1}^{0}, y_{2}^{0}\right)-\varphi\left(y_{1}, y_{2}\right)\right)+2 \alpha(\varepsilon)
$$

and, by integrating w.r.t. $\gamma^{\varepsilon}$, passing to the limit as $\varepsilon \rightarrow 0$ and recalling that both $\eta$ and $\gamma$ are arbitrary, one infers $\Lambda_{g}^{*}\left(y_{1}^{0}\right) \leq \Lambda_{g}\left(y_{1}^{0}, y_{2}^{0}\right)$.

To prove the remaining (converse) inequality, we consider the family of functions $V_{g}^{\varepsilon, \delta}$ regular subsolutions of the Hamilton Jacobi integrodifferential equation associated to $v_{g}^{\varepsilon, \delta}$ i.e. satisfying

$$
\mathcal{U}^{u ; \varepsilon} V_{g}^{\varepsilon, \delta}\left(y_{1}, y_{2}\right)+g\left(y_{1}, y_{2}\right)-V_{g}^{\varepsilon, \delta}\left(y_{1}, y_{2}\right) \geq 0
$$

Then, one easily gets using (18) and (17)

$$
\xi\left(y_{1}^{0}\right)-\alpha(\delta, \varepsilon) \leq \mathcal{U}^{u ; \varepsilon} V_{g}^{\varepsilon, \delta}\left(y_{1}, y_{2}\right)+g\left(y_{1}, y_{2}\right)+\left\|-V_{g}^{\varepsilon, \delta}\left(y_{1}, \cdot\right)\right\|_{\infty}+\left\|V_{g}^{\varepsilon, \delta}\left(y_{1}^{0}, \cdot\right)\right\|_{\infty}
$$


and

$$
\left|V_{g}^{\varepsilon, \delta}\left(y_{1}, y_{2}\right)-V_{g}^{\varepsilon, \delta}\left(y_{1}, y_{2}^{\prime}\right)\right| \leq \alpha(\delta, \varepsilon)
$$

where

$$
\alpha(\delta, \varepsilon):=2 c_{0}\left(1+\frac{1}{\varepsilon}\right) \eta\left(c_{0}\left(1+\frac{1}{\varepsilon}\right) \delta\right)+\omega(\varepsilon) .
$$

The reader is invited to note that $\liminf _{\varepsilon \rightarrow 0} \liminf _{\delta \rightarrow 0} \alpha(\delta, \varepsilon)=\liminf _{\varepsilon \rightarrow 0} \omega(\varepsilon)=0$ to deduce that $\xi\left(y_{1}^{0}\right) \leq \Lambda_{g}^{*}\left(y_{1}^{0}\right)$ which concludes the proof of our theorem.

\subsection{Second Main Result: Support for Asymptotic Optimality}

In most of the interesting cases, the function $\eta$ can be computed explicitly (e.g. if $Q$ has some Lipschitz continuity property). For fixed $\varepsilon>0$, we let

$$
\delta^{\varepsilon}:=\varepsilon^{2} \times \sup \left\{\delta>0: \eta(\delta) \leq \varepsilon^{3}\right\} .
$$

Motivated by the classical case, we let, by abuse of notation, $\xi\left(y_{1}^{0}, y_{2}^{0}\right)=$ $\xi\left(y_{1}^{0}\right)$ and we introduce the one-approximating and two-approximating sets by setting

$\Omega_{g}^{1, n}(x):=\left\{\begin{array}{c}(y, u) \in \mathbb{K} \times U, \text { s.t. } \xi(x)+\eta\left(\left(\xi(x)-\mu^{n}\right)^{\frac{1}{2}}\right) \\ \geq \mathcal{U}^{u ; n^{-1}} V_{g}^{n^{-1}, \delta^{n^{-1}}}(y)+g(y)+\left(V_{g}^{n^{-1}, \delta^{n^{-1}}}(x)-V_{g}^{n^{-1}, \delta^{n^{-1}}}(y)\right)\end{array}\right\}$,

where $\left(\mu^{n}, V_{g}^{n^{-1}, \delta^{n-1}}\right) \in D_{g}^{n^{-1}}(x)$ (defined as in (9) but for the infinitesimal operator $\left.\mathcal{U}^{u ; n^{-1}}\right)$, respectively $\Omega_{g}^{2, n}(x)$ in which $\xi(x)$ is replaced by $v_{g}^{n^{-1}}$ and the usual (inner ant outer) limit sets

$$
\Omega_{g}^{2, \text { in }}(x):=\liminf _{n \rightarrow \infty} \Omega_{g}^{2, n}(x), \Omega_{g}^{2, \text { out }, c l}(x):=\underset{n \geq 1}{\cap} c l\left(\underset{k \geq n}{\cup} \Omega_{g}^{2, k}(x)\right) .
$$

Remark 4.1. A glance at the proof of the main result shows that the value function $\xi$ is the common limit to both $v_{g}^{n^{-1}}(x)$ and such $\mu^{n}$.

We get the following result stating that optimal measures can be obtained as limit of optimal measures for the perturbed problems and, then, the support is included in the outer limit. Conversely, if one takes admissible measures whose support is reasonably close to $\Omega_{g}^{1, n}(x)$, any limit of such sequence provides an optimal measure.

Theorem 4.2. Let us fix some $\left(y_{1}^{0}, y_{2}^{0}\right) \in \mathbb{K}_{1} \times \mathbb{K}_{2}$. 
i. If $\gamma_{n} \in \Theta^{\frac{1}{n}}\left(y_{1}^{0}, y_{2}^{0}\right)$ is optimal, then any limit $\gamma$ is optimal and $\gamma\left(\Omega_{g}^{2, \text { out }, c l}\right)=1$.

ii. If $\gamma_{n} \in \Theta^{\frac{1}{n}}\left(y_{1}^{0}, y_{2}^{0}\right)$ is such that $\gamma_{n}\left(\left(\Omega_{g}^{1, n}(x)\right)^{c}\right)=o\left(\frac{\delta^{n^{-1}}}{n}\right)$, then any limit of $\gamma_{n}$ is optimal.

Proof. For the first assertion, since $\gamma_{n} \in \Theta^{\frac{1}{n}}\left(y_{1}^{0}, y_{2}^{0}\right)$ is optimal, one has

$$
\begin{aligned}
v_{g}^{n^{-1}}\left(y_{1}^{0}, y_{2}^{0}\right) & =\int_{\mathbb{R}^{N+M} \times U}\left[\mathcal{U}^{u ; n^{-1}} V_{g}^{n^{-1}, \delta^{n^{-1}}}(y)+g(y)+\left(V_{g}^{n^{-1}, \delta^{n^{-1}}}(x)-V_{g}^{n^{-1}, \delta^{n^{-1}}}(y)\right)\right] \gamma_{n}(d y d u) \\
& \geq \mu^{n} \gamma_{n}\left(\Omega_{g}^{2, n}(x)\right)+\left[v_{g}^{n^{-1}}(x)+\eta\left(\left(v_{g}^{n^{-1}}(x)-\mu^{n}\right)^{\frac{1}{2}}\right)\right] \gamma_{n}\left(\left(\Omega_{g}^{2, n}(x)\right)^{c}\right) .
\end{aligned}
$$

As in the classical case, one infers that

$$
\gamma_{n}\left(\Omega_{g}^{2, n}(x)\right) \geq \frac{1}{1+\frac{v_{g}^{n^{-1}}(x)-\mu^{n}}{\eta\left(\left(v_{g}^{n^{-1}}(x)-\mu^{n}\right)^{\frac{1}{2}}\right)}} .
$$

Therefore, for every $n \geq n_{0} \geq 1$, one has

$$
\gamma_{n}\left(c l\left(\underset{k \geq n_{0}}{\cup} \Omega_{g}^{2, k}(x)\right)\right) \geq \frac{1}{1+\frac{v_{g}^{n^{-1}}(x)-\mu^{n}}{\eta\left(\left(v_{g}^{n-1}(x)-\mu^{n}\right)^{\frac{1}{2}}\right)}}
$$

and the conclusion follows by letting $n \rightarrow \infty$ (and recalling Remark 4.1).

For the second assertion, one begins with noting that, due to (19), the following inequatity holds $\mathcal{U}^{u ; n^{-1}} V_{g}^{n^{-1}, \delta^{n^{-1}}}(y) \leq \frac{c_{0}(1+n)}{\delta^{n^{-1}}}$. Consequently,

$$
\begin{aligned}
\xi\left(y_{1}^{0}\right) & \leq \lim _{n \rightarrow \infty} \int_{\mathbb{R}^{N+M} \times U}\left[\mathcal{U}^{u ; n^{-1}} V_{g}^{n^{-1}, \delta^{n^{-1}}}(y)+g(y)+\left(V_{g}^{n^{-1}, \delta^{n^{-1}}}(x)-V_{g}^{n^{-1}, \delta^{n^{-1}}}(y)\right)\right] \gamma_{n}(d y d u) \\
& \leq \lim _{n \rightarrow \infty}\left[\xi\left(y_{1}^{0}\right)+\eta\left(\left(\xi\left(y_{1}^{0}\right)-\mu^{n}\right)^{\frac{1}{2}}\right)+\left(\frac{c_{0}(1+n)}{\delta^{n^{-1}}}+3\|g\|_{\infty}\right) \gamma_{n}\left(\left(\Omega_{g}^{1, n}(x)\right)^{c}\right)\right]
\end{aligned}
$$

and the conclusion follows.

\section{References}

1. N. V. Krylov, On the rate of convergence of finite-difference approximations for Bellman's equations with variable coefficients, Probab. Theory Related Fields 117, 1 (2000).

2. V. Gaitsgory, Suboptimization of singularly perturbed control systems, SIAM J. Control and Optim. 30, 1228 (1992). 
3. G. Yin and Q. Zhang, Control of dynamic systems under the influence of singularly perturbed markov chains, Journal of Mathematical Analysis and Applications 216, 343 (1997).

4. E. Fridman, Exact slow-fast decomposition of a class of non-linear singularly perturbed optimal control problems via invariant manifolds, International Journal of Control 72, 1609 (1999).

5. D. Naidu, Singular perturbations and time scales in control theory and applications: an overview, Dynamics of Continuous, Discrete and Impulsive Systems, Series B , 233 (2002).

6. O. L. V. Costa and F. Dufour, Singular perturbation for the discounted continuous control of piecewise deterministic markov processes, Applied Mathematics \&3 Optimization 63, 357 (2010).

7. O. Serea, Characterization of the optimal trajectories for the averaged dynamics associated to singularly perturbed control systems, accepted in Journal of Differential Equations (2014).

8. D. Goreac, Asymptotic control for a class of piecewise deterministic markov processes associated to temperate viruses, SIAM Journal on Control and Optimization 53, 1860 (2015).

9. V. Borkar and V. Gaitsgory, Averaging of singularly perturbed controlled stochastic differential equations, Appl. Math. Optimization 56, 169 (2007).

10. M. H. A. Davis, Piecewise-deterministic Markov-processes - A generalclass of non-diffusion stochastic-models, Journal of the Royal Statistical Society Series B-Methodological 46, 353 (1984).

11. M. H. A. Davis, Control of Piecewise-deterministic processes via discrete-time dynamic-programming, Lect. Notes Control Inf. Sci. 78, 140 (1986).

12. M. H. A. Davis, Markov models and optimization, Monographs on Statistics and Applied Probability, Vol. 49 (Chapman \& Hall, London, 1993).

13. D. Goreac, Viability, Invariance and Reachability for Controlled Piecewise Deterministic Markov Processes Associated to Gene Networks, ESAIM-Control Optimisation and Calculus of Variations 18, 401 (APR 2012).

14. D. Goreac and O.-S. Serea, Linearization Techniques for Controlled Piecewise Deterministic Markov Processes; Application to Zubov's Method, Applied Mathematics and Optimization 66, 209 (2012).

15. V. Borkar and V. Gaitsgory, On existence of limit occupational measures set of a controlled stochastic differential equation, SIAM J. Con- 
trol Optim. 44, 1436 (2005). 